\title{
Arbitrary order fractional differential equations and inclusions with new integro-multipoint boundary conditions
}

\author{
Sara Salem ${ }^{1}$, Ahmed Alsaedi ${ }^{*}$ (D), Bashir Ahmad ${ }^{1}$ and Maryem Al-Yami ${ }^{1}$
}

*Correspondence:

aalsaedi@hotmail.com

${ }^{1}$ Nonlinear Analysis and Applied

Mathematics (NAAM) - Research

Group, Department of Mathematics,

Faculty of Science, King Abdulaziz

University, Jeddah, Saudi Arabia

\begin{abstract}
In this paper, we study a new boundary value problem of arbitrary order fractional differential equations equipped with new integro-multipoint boundary conditions. Existence and uniqueness results for the given problem are obtained by applying the standard tools of fixed point theory. We also extend the problem at hand to its inclusions case and prove an existence result for it by applying a fixed point theorem due to Bohnenblust and Karlin.
\end{abstract}

MSC: 34A08; 34B10; 34B15

Keywords: Caputo fractional derivative; Multi-point; Integral boundary conditions; Existence; Fixed point

\section{Introduction}

Fractional calculus is a branch of mathematical analysis that deals with the derivatives and integrals of arbitrary (non-integer) order. In fact fractional calculus has developed into an important field of research during the last few decades in view of its widespread applications in a variety of disciplines such as physics, chemistry, biology, biophysics, blood flow phenomena, control theory, wave propagation, signal and image processing, viscoelasticity, financial mathematics, economics, etc. For theoretical background of the subject, we refer the reader to the texts [1-4], while the application of fractional calculus can be found, for instance, in [5-12]. An interesting feature of fractional-order operators is their nonlocal nature that accounts for hereditary characteristics of many materials and processes in contrast to the corresponding integer-order differential operators. Nowadays, fractional derivatives appear naturally in the mathematical modeling of dynamical systems involving fractals and chaos.

Boundary value problems of fractional-order differential equations and inclusions supplemented with different types of boundary conditions have recently been investigated by many researchers. Examples include classical, nonlocal, multi-point, periodic/antiperiodic, and integral boundary conditions. The literature on the topic is now much enriched, and it contains a wide variety of results ranging from existence and uniqueness theory to the analytic and numerical methods for solving such problems. Here, it is imperative to mention that nonlocal conditions $[13,14]$ are found to be more practical than

(C) The Author(s) 2018. This article is distributed under the terms of the Creative Commons Attribution 4.0 International License (http://creativecommons.org/licenses/by/4.0/), which permits unrestricted use, distribution, and reproduction in any medium, provided you give appropriate credit to the original author(s) and the source, provide a link to the Creative Commons license, and indicate if changes were made. 
the classical initial/boundary conditions in view of their ability to describe some peculiarities of physical, chemical or other processes happening inside the domain. In the study of blood flow problems, it is not always possible to assume the circular channels. To overcome this issue, integral boundary conditions [15] provide an efficient approach and make it possible to consider channels of arbitrary shape. Such conditions also play an important role in the study of mathematical models for bacterial self-regularization [16] as these conditions help to regularize ill-posed parabolic backward problems in time partial differential equations. Some recent work on the topic can be found in a series of papers [17-37] and the references cited therein.

Motivated by recent works on nonlocal fractional order boundary value problems, we investigate the existence and uniqueness of solutions for the following arbitrary order differential equation:

$$
{ }^{\mathrm{c}} D^{q} x(t)=f(t, x(t)), \quad 0<t<1, m-1<q \leq m, m \geq 2, m \in N,
$$

supplemented with a new type of integro-multipoint boundary conditions of the form:

$$
\left\{\begin{array}{l}
\int_{0}^{1} x(s) d s=\sum_{j=1}^{p} \beta_{j} x\left(\sigma_{j}\right), \quad x^{\prime}(0)=0, x^{\prime \prime}(0)=0, \ldots, x^{(m-2)}(0)=0, \\
a_{1} x(1)+a_{2}{ }^{\mathrm{c}} D^{q-1} x(1)=\sum_{i=1}^{n-2} \alpha_{i} \int_{\xi_{i}}^{\eta_{i}} x(s) d s
\end{array}\right.
$$

where ${ }^{\mathrm{c}} D^{q}$ denotes the Caputo fractional derivative of order $q, f$ is a given continuous function, $0<\sigma_{1}<\sigma_{2}<\cdots<\sigma_{p-1}<\sigma_{p}<\xi_{1}<\eta_{1}<\cdots<\xi_{n-2}<\eta_{n-2}<1$ and $\alpha_{i}, \beta_{j}, a_{1}, a_{2} \in \mathbb{R}$, $i=1,2, \ldots,(n-2), j=1,2, \ldots, p$.

The rest of the paper is organized as follows. Section 2 contains the basic concepts of fractional calculus and an auxiliary lemma. In Sect. 3, we present the existence and uniqueness results for problem (1.1)-(1.2), while Sect. 4 illustrates the results obtained in Sect. 3 with the aid of examples. The classical boundary conditions case of problem (1.1)-(1.2) is discussed in Sect. 5. In the final section, we prove an existence result for the multi-valued analogue of problem (1.1)-(1.2).

\section{Preliminaries}

Before presenting some auxiliary results, let us recall some preliminary concepts of fractional calculus $[1,3]$.

Definition 2.1 Let $g$ be a locally integrable real-valued function on $-\infty \leq a<t<b \leq+\infty$. The Riemann-Liouville fractional integral $I_{a}^{\alpha}$ of order $\alpha \in \mathbb{R}(\alpha>0)$ is defined as

$$
I_{a}^{\alpha} g(t)=\left(g * K_{\alpha}\right)(t)=\frac{1}{\Gamma(\alpha)} \int_{a}^{t}(t-s)^{\alpha-1} g(s) d s,
$$

where $K_{\alpha}(t)=\frac{t^{\alpha-1}}{\Gamma(\alpha)}, \Gamma$ denotes the Euler gamma function.

Definition 2.2 Let $g \in L^{1}[a, b],-\infty \leq a<t<b \leq+\infty$ and $g * K_{m-\alpha} \in W^{m, 1}[a, b], m=$ $[\alpha]+1, \alpha>0$, where $W^{m, 1}[a, b]$ is the Sobolev space defined as

$$
W^{m, 1}[a, b]=\left\{g \in L^{1}[a, b]: \frac{d^{m}}{d t^{m}} g \in L^{1}[a, b]\right\} .
$$


The Riemann-Liouville fractional derivative $D_{a}^{\alpha}$ of order $\alpha>0(m-1<\alpha<m, m \in \mathbb{N})$ is defined as

$$
D_{a}^{\alpha} g(t)=\frac{d^{m}}{d t^{m}} I_{a}^{1-\alpha} g(t)=\frac{1}{\Gamma(m-\alpha)} \frac{d^{m}}{d t^{m}} \int_{a}^{t}(t-s)^{m-1-\alpha} g(s) d s
$$

Definition 2.3 Let $g \in L^{1}[a, b],-\infty \leq a<t<b \leq+\infty$ and $g * K_{m-\alpha} \in W^{m, 1}[a, b], m=[\alpha]$, $\alpha>0$. The Caputo fractional derivative ${ }^{\mathrm{c}} D_{a}^{\alpha}$ of order $\alpha \in \mathbb{R}(m-1<\alpha<m, m \in \mathbb{N})$ is defined as

$$
{ }^{\mathrm{c}} D_{a}^{\alpha} g(t)=D_{a}^{\alpha}\left[g(t)-g(a)-g^{\prime}(a) \frac{(t-a)}{1 !}-\cdots-g^{(m-1)}(a) \frac{(t-a)^{m-1}}{(m-1) !}\right] .
$$

Remark 2.4 If $g \in C^{m}[a, b]$, then the Caputo fractional derivative ${ }^{\mathrm{c}} D_{a}^{\alpha}$ of order $\alpha \in \mathbb{R}$ (m$1<\alpha<m, m \in \mathbb{N}$ ) is defined as

$$
{ }^{\mathrm{c}} D_{a}^{\alpha}[g](t)=I_{a}^{1-\alpha} g^{(m)}(t)=\frac{1}{\Gamma(m-\alpha)} \int_{a}^{t}(t-s)^{m-1-\alpha} g^{(m)}(s) d s .
$$

In the sequel, the Riemann-Liouville fractional integral $I_{a}^{\alpha}$ and the Caputo fractional derivative ${ }^{\mathrm{c}} D_{a}^{\alpha}$ with $a=0$ are respectively denoted by $I^{\alpha}$ and ${ }^{\mathrm{c}} D^{\alpha}$.

Definition 2.5 A function $x \in C^{m}[0,1]$ satisfying problem (1.1)-(1.2) is called its solution on $[0,1]$.

Relative to the linear variant of problem (1.1)-(1.2), we consider the following lemma.

Lemma 2.6 Let $g \in C(0,1) \cap L(0,1)$ and $\Delta=A_{2} A_{3}-A_{1} A_{4} \neq 0$. Then the solution of the linear fractional differential equation ${ }^{\mathrm{c}} D^{q} x(t)=g(t), m-1<q \leq m$, supplemented with the boundary conditions (1.2) is given by

$$
\begin{aligned}
x(t)= & \int_{0}^{t} \frac{(t-s)^{q-1}}{\Gamma(q)} g(s) d s-\lambda_{1}(t)\left[\sum_{i=1}^{n-2} \alpha_{i} \int_{\xi_{i}}^{\eta_{i}}\left(\int_{0}^{s} \frac{(s-u)^{q-1}}{\Gamma(q)} g(u) d u\right) d s\right. \\
& \left.-a_{1} \int_{0}^{1} \frac{(1-s)^{q-1}}{\Gamma(q)} g(s) d s-a_{2} \int_{0}^{1} g(s) d s\right] \\
& -\lambda_{2}(t)\left[\int_{0}^{1}\left(\int_{0}^{s} \frac{(s-u)^{q-1}}{\Gamma(q)} g(u) d u\right) d s-\sum_{j=1}^{p} \beta_{j} \int_{0}^{\sigma_{j}} \frac{\left(\sigma_{j}-s\right)^{q-1}}{\Gamma(q)} g(s) d s\right],
\end{aligned}
$$

where

$$
\begin{aligned}
& \lambda_{1}(t)=\frac{A_{2}-A_{1} t^{m-1}}{\Delta}, \quad \lambda_{2}(t)=\frac{A_{4}-A_{3} t^{m-1}}{\Delta}, \\
& A_{1}=1-\sum_{j=1}^{p} \beta_{j}, \quad A_{2}=\frac{1}{m}-\sum_{j=1}^{p} \beta_{j} \sigma_{j}^{m-1}, \\
& A_{3}=\sum_{i=1}^{n-2} \alpha_{i}\left(\eta_{i}-\xi_{i}\right)-a_{1}, \quad A_{4}=\sum_{i=1}^{n-2} \alpha_{i} \frac{\eta_{i}^{m}-\xi_{i}^{m}}{m}-\left(a_{1}+a_{2} \frac{(m-1) !}{\Gamma(m-q+1)}\right) .
\end{aligned}
$$


Proof It is well known that the general solution of the fractional differential equation ${ }^{\mathrm{c}} D^{q} x(t)=g(t), m-1<q \leq m$, can be written as

$$
x(t)=\int_{0}^{t} \frac{(t-s)^{q-1}}{\Gamma(q)} g(s) d s-c_{0}-c_{1} t-\cdots-c_{m-2} t^{m-2}-c_{m-1} t^{m-1},
$$

where $c_{0}, c_{1}, c_{2}, \ldots, c_{m-1}$ are unknown arbitrary constants. Using the boundary conditions $x^{\prime}(0)=0, \ldots, x^{(m-2)}(0)=0$ in $(2.3)$, we get $c_{1}=0, \ldots, c_{m-2}=0$. Thus (2.3) takes the form

$$
x(t)=\int_{0}^{t} \frac{(t-s)^{q-1}}{\Gamma(q)} g(s) d s-c_{0}-c_{m-1} t^{m-1} .
$$

Making use of the first and last conditions of (1.2) in (2.4), we find a system of algebraic equations in $c_{0}$ and $c_{m-1}$ given by

$$
\left\{\begin{array}{l}
A_{1} c_{0}+A_{2} c_{m-1}=\phi_{1}, \\
A_{3} c_{0}+A_{4} c_{m-1}=\phi_{2},
\end{array}\right.
$$

where $A_{1}, A_{2}, A_{3}, A_{4}$ are given by (2.2), and

$$
\begin{aligned}
& \phi_{1}=\int_{0}^{1}\left(\int_{0}^{s} \frac{(s-u)^{q-1}}{\Gamma(q)} g(u) d u\right) d s-\sum_{j=1}^{p} \beta_{j} \int_{0}^{\sigma_{j}} \frac{\left(\sigma_{j}-s\right)^{q-1}}{\Gamma(q)} g(s) d s, \\
& \phi_{2}=\sum_{i=1}^{n-2} \alpha_{i} \int_{\xi_{i}}^{\eta_{i}}\left(\int_{0}^{s} \frac{(s-u)^{q-1}}{\Gamma(q)} g(u) d u\right) d s-a_{1} \int_{0}^{1} \frac{(1-s)^{q-1}}{\Gamma(q)} g(s) d s-a_{2} \int_{0}^{1} g(s) d s .
\end{aligned}
$$

Solving system (2.5) for $c_{0}$ and $c_{m-1}$ together with notations (2.2), we get

$$
c_{0}=\frac{1}{\Delta}\left(A_{2} \phi_{2}-\phi_{1} A_{4}\right), \quad c_{m-1}=\frac{1}{\Delta}\left(A_{3} \phi_{1}-A_{1} \phi_{2}\right),
$$

which, on substituting in (2.4), yields solution (2.1). We can establish the converse of the lemma by direct computation. This completes the proof.

\section{Existence and uniqueness results}

Denote by $C([0,1], \mathbb{R})$ the Banach space of all continuous functions from $[0,1] \rightarrow \mathbb{R}$ endowed with the norm $\|x\|=\sup \{|x(t)|, t \in[0,1]\}$. In view of Lemma 2.6, we transform problem (1.1)-(1.2) into a fixed point problem as $x=\varphi x$, where the operator $\varphi: C([0,1], \mathbb{R}) \rightarrow$ $C([0,1], \mathbb{R})$ is defined by

$$
\begin{aligned}
(\varphi x)(t)= & \int_{0}^{t} \frac{(t-s)^{q-1}}{\Gamma(q)} f(s, x(s)) d s-\lambda_{1}(t)\left[\sum_{i=1}^{n-2} \alpha_{i} \int_{\xi_{i}}^{\eta_{i}}\left(\int_{0}^{s} \frac{(s-u)^{q-1}}{\Gamma(q)} f(u, x(u)) d u\right) d s\right. \\
& \left.-a_{1} \int_{0}^{1} \frac{(1-s)^{q-1}}{\Gamma(q)} f(s, x(s)) d s-a_{2} \int_{0}^{1} f(s, x(s)) d s\right] \\
& -\lambda_{2}(t)\left[\int_{0}^{1}\left(\int_{0}^{s} \frac{(s-u)^{q-1}}{\Gamma(q)} f(u, x(u)) d u\right) d s\right. \\
& \left.-\sum_{j=1}^{p} \beta_{j} \int_{0}^{\sigma_{j}} \frac{\left(\sigma_{j}-s\right)^{q-1}}{\Gamma(q)} f(s, x(s)) d s\right], \quad t \in[0,1] .
\end{aligned}
$$


For the sake of computational convenience, let us set

$$
\begin{aligned}
\Lambda= & \frac{1}{\Gamma(q+1)}+\bar{\lambda}_{1}\left(\sum_{i=1}^{n-2} \frac{\left|\alpha_{i}\left(\eta_{i}^{q+1}-\xi_{i}^{q+1}\right)\right|}{\Gamma(q+2)}+\frac{\left|a_{1}\right|}{\Gamma(q+1)}+\left|a_{2}\right|\right) \\
& +\bar{\lambda}_{2}\left(\frac{1}{\Gamma(q+2)}+\sum_{j=1}^{p} \frac{\left|\beta_{j} \sigma_{j}^{q}\right|}{\Gamma(q+1)}\right), \\
\Lambda_{1}= & \Lambda-\frac{1}{\Gamma(q+1)}=\bar{\lambda}_{1}\left(\sum_{i=1}^{n-2} \frac{\left|\alpha_{i}\left(\eta_{i}^{q+1}-\xi_{i}^{q+1}\right)\right|}{\Gamma(q+2)}+\frac{\left|a_{1}\right|}{\Gamma(q+1)}+\left|a_{2}\right|\right) \\
& +\bar{\lambda}_{2}\left(\frac{1}{\Gamma(q+2)}+\sum_{j=1}^{p} \frac{\left|\beta_{j} \sigma_{j}^{q}\right|}{\Gamma(q+1)}\right)
\end{aligned}
$$

where

$$
\bar{\lambda}_{1}=\max _{t \in[0,1]}\left|\lambda_{1}(t)\right|=\frac{1}{|\Delta|}\left(\left|A_{2}\right|+\left|A_{1}\right|\right), \quad \bar{\lambda}_{2}=\max _{t \in[0,1]}\left|\lambda_{2}(t)\right|=\frac{1}{|\Delta|}\left(\left|A_{4}\right|+\left|A_{3}\right|\right) .
$$

Now we are in a position to present our main results concerning the existence and uniqueness of solutions of problem (1.1)-(1.2). Our first result is based on a nonlinear alternative of Leray-Schauder type.

Lemma 3.1 (Nonlinear alternative for single-valued maps $[38])$ Let $C([0,1], \mathbb{R})$ be a closed and convex subset of Banach space $E$ and $U$ be an open subset of $C([0,1], \mathbb{R})$ with $0 \in U$. Suppose that $F: \bar{U} \rightarrow C([0,1], \mathbb{R})$ is a continuous, compact (that is, $F(\bar{U})$ is a relatively compact subset of $C([0,1], \mathbb{R}))$ map. Then either (i) $F$ has a fixed point in $\bar{U}$, or (ii) there are $u \in \partial U$ (the boundary of $U$ in $C([0,1], \mathbb{R})$ ) and $\lambda \in(0,1)$ with $u=\lambda F(u)$.

Theorem 3.2 Let $f:[0,1] \times \mathbb{R} \rightarrow \mathbb{R}$ be a continuous function such that the following conditions hold:

$\left(\mathrm{A}_{1}\right)$ There exist a function $p \in C\left([0,1], \mathbb{R}^{+}\right)$and a nondecreasing function $v: \mathbb{R}^{+} \rightarrow \mathbb{R}^{+}$ such that $|f(t, x)| \leq p(t) v(\|x\|)$ for all $(t, x) \in[0,1] \times \mathbb{R} ;$

$\left(\mathrm{A}_{2}\right)$ There exists a positive constant $M>0$ such that $\frac{M}{\|p\| v(M) \Lambda}>1$, where $\|p\|=$ $\max _{t \in[0,1]}|p(t)|$ and $\Lambda$ is given by (3.2).

Then problem (1.1)-(1.2) has at least one solution on $[0,1]$.

Proof We complete the proof in several steps. First of all, we show that the operator $\varphi: C([0,1], \mathbb{R}) \rightarrow C([0,1], \mathbb{R})$ denoted by (3.1) maps bounded sets into bounded sets in $C([0,1], \mathbb{R})$. For a positive number $r$, let $B_{r}=\{x \in C([0,1], \mathbb{R}):\|x\| \leq r\}$ be a bounded set in $C([0,1], \mathbb{R})$. Then, by assumption $\left(\mathrm{A}_{1}\right)$, we obtain

$$
\begin{aligned}
|(\varphi x)(t)| \leq & \max _{t \in[0,1]}\left\{\int_{0}^{t} \frac{(t-s)^{q-1}}{\Gamma(q)}|f(s, x(s))| d s\right. \\
& +\left|\lambda_{1}(t)\right|\left[\sum_{i=1}^{n-2}\left|\alpha_{i}\right| \int_{\xi_{i}}^{\eta_{i}}\left(\int_{0}^{s} \frac{(s-u)^{q-1}}{\Gamma(q)}|f(u, x(u))| d u\right) d s\right. \\
& \left.+\left|a_{1}\right| \int_{0}^{1} \frac{(1-s)^{q-1}}{\Gamma(q)}|f(s, x(s))| d s+\left|a_{2}\right| \int_{0}^{1}|f(s, x(s))| d s\right]
\end{aligned}
$$




$$
\begin{aligned}
& +\left|\lambda_{2}(t)\right|\left[\int_{0}^{1}\left(\int_{0}^{s} \frac{(s-u)^{q-1}}{\Gamma(q)}|f(u, x(u))| d u\right) d s\right. \\
& \left.\left.+\sum_{j=1}^{p}\left|\beta_{j}\right| \int_{0}^{\sigma_{j}} \frac{\left(\sigma_{j}-s\right)^{q-1}}{\Gamma(q)}|f(s, x(s))| d s\right]\right\} \\
& \leq\|p\| v(\|x\|)\left[\frac{1}{\Gamma(q+1)}+\bar{\lambda}_{1}\left(\sum_{i=1}^{n-2} \frac{\left|\alpha_{i}\left(\eta_{i}^{q+1}-\xi_{i}^{q+1}\right)\right|}{\Gamma(q+2)}+\frac{\left|a_{1}\right|}{\Gamma(q+1)}+\left|a_{2}\right|\right)\right. \\
& \left.+\bar{\lambda}_{2}\left(\frac{1}{\Gamma(q+2)}+\sum_{j=1}^{p} \frac{\left|\beta_{j} \sigma_{j}^{q}\right|}{\Gamma(q+1)}\right)\right] \\
& \leq\|p\| v(\|x\|) \Lambda,
\end{aligned}
$$

where we have used (3.2). Next we show that $\varphi$ maps bounded sets into equicontinuous sets of $C([0,1], \mathbb{R})$. Let $0<\tau_{1}<\tau_{2}<1$ and $x \in B_{r}$, where $B_{r}$ is a bounded set of $C([0,1], \mathbb{R})$. Then we have

$$
\begin{aligned}
\left|\varphi x\left(\tau_{2}\right)-\varphi x\left(\tau_{1}\right)\right| & \left|\int_{0}^{\tau_{1}} \frac{\left(\tau_{2}-s\right)^{q-1}-\left(\tau_{1}-s\right)^{q-1}}{\Gamma(q)} f(s, x(s)) d s+\int_{\tau_{1}}^{\tau_{2}} \frac{\left(\tau_{2}-s\right)^{q-1}}{\Gamma(q)} f(s, x(s)) d s\right| \\
& +\frac{\left|\tau_{2}^{m-1}-\tau_{1}^{m-1}\right|}{|\Delta|}\left[| A _ { 1 } | \left(\sum_{i=1}^{n-2}\left|\alpha_{i}\right| \int_{\xi_{i}}^{\eta_{i}}\left(\int_{0}^{s} \frac{(s-u)^{q-1}}{\Gamma(q)}|f(u, x(u))| d u\right) d s\right.\right. \\
& \left.+\left|a_{1}\right| \int_{0}^{1} \frac{(1-s)^{q-1}}{\Gamma(q)}|f(s, x(s))| d s+\left|a_{2}\right| \int_{0}^{1}|f(s, x(s))| d s\right) \\
& +\left|A_{3}\right|\left(\int_{0}^{1}\left(\int_{0}^{s} \frac{(s-u)^{q-1}}{\Gamma(q)}|f(u, x(u))| d u\right) d s\right. \\
& \left.\left.+\sum_{j=1}^{p}\left|\beta_{j}\right| \int_{0}^{\sigma_{j}} \frac{\left(\sigma_{j}-s\right)^{q-1}}{\Gamma(q)}|f(s, x(s))| d s\right)\right] \\
\leq & \|p\| v(r)\left[\frac{2\left(\tau_{2}-\tau_{1}\right)^{q}+\left|\tau_{2}^{q}-\tau_{1}^{q}\right|}{\Gamma(q+1)}\right. \\
& +\frac{\left|\tau_{2}^{m-1}-\tau_{1}^{m-1}\right|}{|\Delta|}\left\{\left|A_{1}\right|\left(\sum_{i=1}^{n-2} \frac{\left|\alpha_{i}\left(\eta_{i}^{q+1}-\xi_{i}^{q+1}\right)\right|}{\Gamma(q+2)}+\frac{\left|a_{1}\right|}{\Gamma(q+1)}+\left|a_{2}\right|\right)\right. \\
& \left.\left.+\left|A_{3}\right|\left(\frac{1}{\Gamma(q+2)}+\sum_{j=1}^{p} \frac{\left|\beta_{j} \sigma_{j}^{q}\right|}{\Gamma(q+1)}\right)\right\}\right] .
\end{aligned}
$$

Obviously the right-hand side of the above inequality tends to zero independently of $x \in B_{r}$ as $\tau_{2}-\tau_{1} \rightarrow 0$. In view of the foregoing arguments, it follows by the Arzela-Ascoli theorem that $\varphi: C([0,1], \mathbb{R}) \rightarrow C([0,1], \mathbb{R})$ is completely continuous.

The result will follow from (Lemma 3.1) once it is established that the set of all solutions to equations $x=\lambda \varphi x$ for $\lambda \in[0,1]$ is bounded. Let $x$ be a solution of problem (1.1)-(1.2). Then, for $t \in[0,1]$, as in the first step, we can find that

$$
\|x\|=\|\lambda(\varphi x)(t)\| \leq\|p\| v(\|x\|) \Lambda,
$$


which can alternatively be written as

$$
\frac{\|x\|}{\|p\| v(\|x\|) \Lambda} \leq 1
$$

By condition $\left(\mathrm{A}_{2}\right)$, there exists $M>0$ such that $\|x\| \neq M$. Let us set $\mathcal{U}=\{x \in C([0,1], \mathbb{R})$ : $\|x\|<M\}$ and note that the operator $\varphi: \overline{\mathcal{U}} \rightarrow C([0,1], \mathbb{R})$ is continuous and completely continuous. From the choice of $\mathcal{U}$, there is no $x \in \partial \mathcal{U}$ such that $x=\lambda \varphi(x)$ for some $\lambda \in(0,1)$. Consequently, we deduce by Lemma 3.1 that $\varphi$ has a fixed point $x \in \overline{\mathcal{U}}$, which is a solution of problem (1.1)-(1.2). This completes the proof.

In the following result, we prove the existence of solutions for problem (1.1)-(1.2) by applying Krasnoselskii's fixed point theorem.

Lemma 3.3 (Krasnoselskii's fixed point theorem [39]) Let $\mathcal{V}$ be a bounded, closed, convex, and nonempty subset of a Banach space $\mathcal{W}$. Let $G_{1}, G_{2}$ be the operators such that (a) $G_{1} v_{1}+$ $G_{2} v_{2} \in \mathcal{V}$ whenever $v_{1}, v_{2} \in \mathcal{V}$; (b) $G_{1}$ is compact and continuous; (c) $G_{2}$ is a contraction mapping. Then there exists $v \in \mathcal{V}$ such that $v=G_{1} v+G_{2} v$.

Theorem 3.4 Let $f:[0,1] \times \mathbb{R} \rightarrow \mathbb{R}$ be a continuous function satisfying the conditions:

$\left(\mathrm{A}_{3}\right)|f(t, x)-f(t, y)| \leq L|x-y|$ for all $t \in[0,1], L>0, x, y \in \mathbb{R}$;

$\left(\mathrm{A}_{4}\right)$ There exists a function $\mu \in C\left([0,1], \mathbb{R}^{+}\right)$with $\|\mu\|=\max _{t \in[0,1]}|\mu(t)|$ such that $|f(t, x)| \leq \mu(t)$ for all $(t, x) \in[0,1] \times \mathbb{R}$.

Then there exists at least one solution for problem (1.1)-(1.2) on $[0,1]$ provided that $L \Lambda_{1}<$ 1 , where $\Lambda_{1}$ is given by (3.3).

Proof By assumption ( $\left.\mathrm{A}_{4}\right)$, we can fix $r \geq \Lambda\|\mu\|$, where $\Lambda$ is given by (3.2), and consider the closed set $B_{r}=\left\{x \in C\left([0,1], \mathbb{R}^{+}\right):\|x\| \leq r\right\}$. We define the operators $P$ and $Q$ on $B_{r}$ as

$$
\begin{aligned}
(P x)(t)= & \int_{0}^{t} \frac{(t-s)^{q-1}}{\Gamma(q)} f(s, x(s)) d s, \quad t \in[0,1], \\
(Q x)(t)= & -\lambda_{1}(t)\left[\sum_{i=1}^{n-2} \alpha_{i} \int_{\xi_{i}}^{\eta_{i}}\left(\int_{0}^{s} \frac{(s-u)^{q-1}}{\Gamma(q)} f(u, x(u)) d u\right) d s\right. \\
& \left.-a_{1} \int_{0}^{1} \frac{(1-s)^{q-1}}{\Gamma(q)} f(s, x(s)) d s-a_{2} \int_{0}^{1} f(s, x(s)) d s\right] \\
& -\lambda_{2}(t)\left[\int_{0}^{1}\left(\int_{0}^{s} \frac{(s-u)^{q-1}}{\Gamma(q)} f(u, x(u)) d u\right) d s\right. \\
& \left.-\sum_{j=1}^{p} \beta_{j} \int_{0}^{\sigma_{j}} \frac{\left(\sigma_{j}-s\right)^{q-1}}{\Gamma(q)} f(s, x(s)) d s\right], \quad t \in[0,1] .
\end{aligned}
$$

Now we verify the assumptions of Lemma 3.3. For $x, y \in B_{r}$, we have

$$
\begin{aligned}
\|P x+Q y\| & \leq \sup _{t \in[0,1]}|(P x)(t)+(Q y)(t)| \\
& \leq \sup _{t \in[0,1]}\left\{\int_{0}^{t} \frac{(t-s)^{q-1}}{\Gamma(q)}|f(s, x(s))| d s\right.
\end{aligned}
$$




$$
\begin{aligned}
& +\left|\lambda_{1}(t)\right|\left[\sum_{i=1}^{n-2}\left|\alpha_{i}\right| \int_{\xi_{i}}^{\eta_{i}}\left(\int_{0}^{s} \frac{(s-u)^{q-1}}{\Gamma(q)}|f(u, x(u))| d u\right) d s\right. \\
& \left.+\left|a_{1}\right| \int_{0}^{1} \frac{(1-s)^{q-1}}{\Gamma(q)}|f(s, x(s))| d s+\left|a_{2}\right| \int_{0}^{1}|f(s, x(s))| d s\right] \\
& +\left|\lambda_{2}(t)\right|\left[\int_{0}^{1}\left(\int_{0}^{s} \frac{(s-u)^{q-1}}{\Gamma(q)}|f(u, x(u))| d u\right) d s\right. \\
& \left.\left.+\sum_{j=1}^{p}\left|\beta_{j}\right| \int_{0}^{\sigma_{j}} \frac{\left(\sigma_{j}-s\right)^{q-1}}{\Gamma(q)}|f(s, x(s))| d s\right]\right\} \\
& \leq\|\mu\|\left[\frac{1}{\Gamma(q+1)}+\bar{\lambda}_{1}\left(\sum_{i=1}^{n-2} \frac{\left|\alpha_{i}\left(\eta_{i}^{q+1}-\xi_{i}^{q+1}\right)\right|}{\Gamma(q+2)}+\frac{\left|a_{1}\right|}{\Gamma(q+1)}+\left|a_{2}\right|\right)\right. \\
& \left.+\bar{\lambda}_{2}\left(\frac{1}{\Gamma(q+2)}+\sum_{j=1}^{p} \frac{\left|\beta_{j} \sigma_{j}^{q}\right|}{\Gamma(q+1)}\right)\right] \\
& =\Lambda\|\mu\| \leq r,
\end{aligned}
$$

where we have used $\left(\mathrm{A}_{4}\right)$. This shows that $P x+Q y \in B_{r}$.

Next we establish that $Q$ is a contraction mapping. For $x, y \in C([0,1], \mathbb{R})$ and for each $t \in[0,1]$, we obtain

$$
\begin{aligned}
\|Q x-Q y\| & =\sup _{t \in[0, t]}\left\{| \lambda _ { 1 } ( t ) | \left[\sum_{i=1}^{n-2}\left|\alpha_{i}\right| \int_{\xi_{i}}^{\eta_{i}}\left(\int_{0}^{s} \frac{(s-u)^{q-1}}{\Gamma(q)}|f(u, x(u))-f(u, y(u))| d u\right) d s\right.\right. \\
& \left.+\left|a_{1}\right| \int_{0}^{1} \frac{(1-s)^{q-1}}{\Gamma(q)}|f(s, x(s))-f(s, y(s))| d s+\left|a_{2}\right| \int_{0}^{1}|f(s, x(s))-f(s, y(s))| d s\right] \\
& +\left|\lambda_{2}(t)\right|\left[\int_{0}^{1}\left(\int_{0}^{s} \frac{(s-u)^{q-1}}{\Gamma(q)}|f(u, x(u))-f(u, y(u))| d u\right) d s\right. \\
& \left.\left.+\sum_{j=1}^{p}\left|\beta_{j}\right| \int_{0}^{\sigma_{j}} \frac{\left(\sigma_{j}-s\right)^{q-1}}{\Gamma(q)}|f(s, x(s))-f(s, y(s))| d s\right]\right\} \\
\leq & L\left[\bar{\lambda}_{1}\left(\sum_{i=1}^{n-2} \frac{\left|\alpha_{i}\left(\eta_{i}^{q+1}-\xi_{i}^{q+1}\right)\right|}{\Gamma(q+2)}+\frac{\left|a_{1}\right|}{\Gamma(q+1)}+\left|a_{2}\right|\right)\right. \\
& \left.+\bar{\lambda}_{2}\left(\frac{1}{\Gamma(q+2)}+\sum_{j=1}^{p} \frac{\left|\beta_{j} \sigma_{j}^{q}\right|}{\Gamma(q+1)}\right)\right]\|x-y\| \\
\leq & L \Lambda_{1}\|x-y\|,
\end{aligned}
$$

which shows that the operator $Q$ is a contraction in view of assumption $L \Lambda_{1}<1$.

Notice that the continuity of $f$ implies that the operator $P$ is continuous. Also, $P$ is uniformly bounded on $B_{r}$ as

$$
\|P x\| \leq \sup _{t \in[0,1]}\left\{\int_{0}^{t} \frac{(t-s)^{q-1}}{\Gamma(q)}|f(s, x(s))| d s\right\} \leq \frac{\|\mu\|}{\Gamma(q+1)} .
$$


Finally we show the compactness of the operator $P$. By assumption $\left(\mathrm{A}_{3}\right)$, we define $\max _{(t, x) \in[0,1] \times B_{r}}|f(t, x)|=f_{1}$. Then, for $0<t_{2}<t_{1}<1$, we have

$$
\begin{aligned}
& \left|P x\left(t_{1}\right)-P x\left(t_{2}\right)\right| \\
& \quad=\left|\int_{0}^{t_{2}} \frac{\left(\left(t_{1}-s\right)^{q-1}-\left(t_{2}-s\right)^{q-1}\right)}{\Gamma(q)} f(s, x(s)) d s+\int_{t_{2}}^{t_{1}} \frac{\left(t_{1}-s\right)^{q-1}}{\Gamma(q)} f(s, x(s)) d s\right| \\
& \quad \leq \frac{f_{1}}{\Gamma(q+1)}\left[2\left(t_{1}-t_{2}\right)^{q}+\left|t_{1}^{q}-t_{2}^{q}\right|\right] \rightarrow 0 \quad \text { as }\left(t_{1}-t_{2}\right) \rightarrow 0, \text { independent of } x \in B_{r} .
\end{aligned}
$$

Thus the operator $P$ is relatively compact on $B_{r}$. Hence, by the Arzela-Ascoli theorem, we deduce that $P$ is compact on $B_{r}$. As the hypotheses of Lemma 3.3 are satisfied, so it follows by its conclusion that problem (1.1)-(1.2) has at least one solution on $[0,1]$. The proof is completed.

Finally we prove a uniqueness result for problem (1.1)-(1.2).

Theorem 3.5 Let $f:[0,1] \times \mathbb{R} \rightarrow \mathbb{R}$ be a continuous function satisfying assumption $\left(A_{3}\right)$. Then there exists a unique solution for problem $(1.1)-(1.2)$ on $[0,1]$ if $L \Lambda<1$, where $\Lambda$ is given by (3.2).

Proof We define $B_{r}=\{x \in C([0,1], \mathbb{R}):\|x\| \leq r\}$, where $\max _{t \in[0,1]}|f(t, 0)|=M<\infty$ and $r \geq \frac{M \Lambda}{1-L \Lambda}$, and show that $\varphi B_{r} \subset B_{r}$. For $x \in B_{r}$, observe that

$$
\begin{aligned}
|f(t, x)| & =|f(t, x)-f(t, 0)+f(t, 0)| \leq|f(t, x)-f(t, 0)|+|f(t, 0)| \\
& \leq L|x-0|+|f(t, 0)| \leq L\|x\|+M \leq L r+M .
\end{aligned}
$$

Then, as in the proof of Theorem 3.2, one can obtain that $\|\varphi x\| \leq(L r+M) \Lambda \leq r$, which implies that $\|\varphi x\| \leq r$ for any $x \in B_{r}$. Hence $\varphi B_{r} \subset B_{r}$.

In order to show that the operator $\varphi$ is a contraction, let $x, y \in C([0,1], \mathbb{R})$. Then, for each $t \in[0,1]$, we obtain

$$
\begin{aligned}
\|\varphi x-\varphi y\| \leq & \max _{t \in[0,1]} \mid \int_{0}^{t} \frac{(t-s)^{q-1}}{\Gamma(q)}[f(s, x(s))-f(s, y(s))] d s \\
& +\lambda_{1}(t)\left[\sum_{i=1}^{(n-2)} \alpha_{i} \int_{\xi_{i}}^{\eta_{i}}\left(\int_{0}^{s} \frac{(s-u)^{q-1}}{\Gamma(q)}[f(u, x(u))-f(u, y(u))] d u\right) d s\right. \\
& -a_{1} \int_{0}^{1} \frac{(1-s)^{q-1}}{\Gamma(q)}[f(s, x(s))-f(s, y(s))] d s \\
& \left.-a_{2} \int_{0}^{1}[f(s, x(s))-f(s, y(s))] d s\right] \\
& +\lambda_{2}(t)\left[\int_{0}^{1}\left(\int_{0}^{s} \frac{(s-u)^{q-1}}{\Gamma(q)}[f(u, x(u))-f(u, y(u))] d u\right) d s\right. \\
& \left.+\sum_{j=1}^{p} \beta_{j} \int_{0}^{\sigma_{j}} \frac{\left(\sigma_{j}-s\right)^{q-1}}{\Gamma(q)}[f(s, x(s))-f(s, y(s))] d s\right] \mid
\end{aligned}
$$




$$
\begin{aligned}
\leq & L\left[\frac{1}{\Gamma(q+1)}+\bar{\lambda}_{1}\left(\sum_{i=1}^{n-2} \frac{\left|\alpha_{i}\left(\eta_{i}^{q+1}-\xi_{i}^{q+1}\right)\right|}{\Gamma(q+2)}+\frac{\left|a_{1}\right|}{\Gamma(q+1)}+\left|a_{2}\right|\right)\right. \\
& \left.+\bar{\lambda}_{2}\left(\frac{1}{\Gamma(q+2)}+\sum_{j=1}^{p} \frac{\left|\beta_{j} \sigma_{j}^{q}\right|}{\Gamma(q+1)}\right)\right]\|x-y\| \\
= & L \Lambda\|x-y\|,
\end{aligned}
$$

which shows that the operator $\varphi$ is a contraction by the given assumption $\Lambda L<1$. Hence, by the contraction mapping principle (Banach fixed point theorem), we deduce that problem (1.1)-(1.2) has a unique solution on $[0,1]$. This completes the proof.

\section{Examples}

Consider the following fractional order differential equation:

$$
{ }^{\mathrm{c}} D^{\frac{5}{2}} x(t)=f(t, x(t)), \quad t \in[0,1],
$$

subject to the multi-point and multi-strip boundary conditions

$$
\begin{aligned}
& \int_{0}^{1} x(s) d s=\sum_{j=1}^{3} \beta_{j} x\left(\sigma_{j}\right), \quad x^{\prime}(0)=0, \\
& \frac{1}{2} x(1)+{ }^{\mathrm{c}} D^{q-1} x(1)=\sum_{i=1}^{3} \alpha_{i} \int_{\xi_{i}}^{\eta_{i}} x(s) d s,
\end{aligned}
$$

where $q=\frac{5}{2}, m=3, a_{1}=\frac{1}{2}, a_{2}=1, \sigma_{1}=\frac{1}{28}, \sigma_{2}=\frac{1}{21}, \sigma_{3}=\frac{1}{14}, \xi_{1}=\frac{1}{9}, \xi_{2}=\frac{3}{9}, \xi_{3}=\frac{5}{9}, \eta_{1}=\frac{2}{9}$, $\eta_{2}=\frac{4}{9}, \eta_{3}=\frac{6}{9}, \alpha_{1}=\frac{1}{15}, \alpha_{2}=\frac{1}{10}, \alpha_{3}=\frac{1}{5}, \beta_{1}=\frac{1}{18}, \beta_{2}=\frac{1}{9}, \beta_{3}=\frac{1}{6}$, and $f(t, x)$ will be fixed later. Using the given values, we find that $\Lambda \approx 1.359267$ and $\Lambda_{1} \approx 1.058366$, where $\Lambda$ and $\Lambda_{1}$ are respectively given by (3.2) and (3.3).

To demonstrate the application of Theorem (3.2), we take

$$
f(t, x(t))=\frac{1}{\sqrt{4+t^{2}}}\left(\sin x+\frac{1}{2}+\frac{|x|}{2(1+|x|)}\right), \quad t \in[0,1]
$$

in problem (4.1)-(4.2) and note that

$$
|f(t, x)| \leq \frac{1}{\sqrt{4+t^{2}}}(1+\|x\|) .
$$

Here $p(t)=\frac{1}{\sqrt{4+t^{2}}}$ with $\|p\|=\frac{1}{2}$ and $\psi(\|x\|)=1+\|x\|(\psi(M)=1+M)$. By condition $\left(\mathrm{A}_{2}\right)$, that is, $\frac{M}{\|p\| \psi(M) \Lambda}>1$, we find that $M>2.121425$. Thus all the assumptions of Theorem 3.2 are satisfied. Hence, there exists at least one solution for problem (4.1)-(4.2) with $f(t, x(t))$ given by $(4.3)$ on $[0,1]$.

Next we illustrate Theorems 3.4 and 3.5 by taking

$$
f(t, x(t))=\frac{\left(\sin x+\tan ^{-1} x\right)}{\sqrt{\left(t^{2}+25\right)}}+\left(1+t^{2}\right) e^{t}, \quad 0<t<1,
$$


in problem (4.1)-(4.2), which satisfies the Lipschitz conditions $\left(\mathrm{A}_{3}\right)$ with $L=2 / 5$, that is,

$$
|f(t, x)-f(t, y)| \leq \frac{2}{5}\|x-y\|
$$

and

$$
\|f(t, x)\| \leq \frac{(1+\pi / 2)}{\sqrt{t^{2}+25}}+\left(1+t^{2}\right) e^{t}=\mu(t)
$$

Observe that $L \Lambda_{1} \approx 0.423346<1$ and $L \Lambda \approx 0.543707<1$. Clearly all the assumptions of Theorem 3.4 are satisfied. Hence, by the conclusions of Theorem 3.4, we deduce that there exists at least one solution for problem (4.1)-(4.2) with $f(t, x(t))$ given by $(4.4)$ on $[0,1]$.

Also the hypothesis of Theorem 3.5 holds, which implies that there exists a unique solution for problem (4.1)-(4.2) with $f(t, x(t))$ given by $(4.4)$ on $[0,1]$.

\section{Classical boundary conditions case}

Replacing ${ }^{\mathrm{c}} D^{q-1} x(1)$ with $x^{\prime}(1)$ in the boundary conditions (1.2), we get

$$
\begin{aligned}
& \int_{0}^{1} x(s) d s=\sum_{j=1}^{p} \beta_{j} x\left(\sigma_{j}\right), \quad x^{\prime}(0)=0, x^{\prime \prime}(0)=0, \ldots, x^{(m-2)}(0)=0, \\
& a_{1} x(1)+a_{2} x^{\prime}(1)=\sum_{i=1}^{n-2} \alpha_{i} \int_{\xi_{i}}^{\eta_{i}} x(s) d s, \quad 0<\sigma_{j}<\xi_{i}<\eta_{i}<1 .
\end{aligned}
$$

The fixed point problem associated with the fractional differential equation (1.1) and the boundary conditions (5.1) is $x=\widehat{F} x$, where the operator $\widehat{F}: C([0,1], \mathbb{R}) \rightarrow C([0,1], \mathbb{R})$ is modified to the one given by

$$
\begin{aligned}
(\widehat{F} x)(t)= & \int_{0}^{t} \frac{(t-s)^{q-1}}{\Gamma(q)} f(s, x(s)) d s \\
& -\lambda_{1}(t)\left[\sum_{i=1}^{n-2} \alpha_{i} \int_{\xi_{i}}^{\eta_{i}}\left(\int_{0}^{s} \frac{(s-u)^{q-1}}{\Gamma(q)} f(u, x(u)) d u\right) d s\right. \\
& \left.-\int_{0}^{1}\left(a_{1} \frac{(1-s)^{q-1}}{\Gamma(q)}+a_{2} \frac{(1-s)^{q-2}}{\Gamma(q-1)}\right) f(s, x(s)) d s\right] \\
& -\widehat{\lambda}_{2}(t)\left[\int_{0}^{1}\left(\int_{0}^{s} \frac{(s-u)^{q-1}}{\Gamma(q)} f(u, x(u)) d u\right) d s\right. \\
& \left.-\sum_{j=1}^{p} \beta_{j} \int_{0}^{\sigma_{j}} \frac{\left(\sigma_{j}-s\right)^{q-1}}{\Gamma(q)} f(s, x(s)) d s\right], \quad t \in[0,1],
\end{aligned}
$$

where

$$
\widehat{\lambda}_{2}(t)=\frac{\widehat{A}_{4}-A_{3} t^{m-1}}{\Delta}, \quad \widehat{A}_{4}=\sum_{i=1}^{n-2} \alpha_{i} \frac{\eta_{i}^{m}-\xi_{i}^{m}}{m}-\left(a_{1}+a_{2}(m-1)\right) .
$$


Furthermore, we have

$$
\begin{aligned}
\widehat{\Lambda}= & \frac{1}{\Gamma(q+1)}\left[1+\bar{\lambda}_{1}\left(\sum_{i=1}^{n-2} \frac{\left|\alpha_{i}\left(\eta_{i}^{q+1}-\xi_{i}^{q+1}\right)\right|}{q+1}+\left|a_{1}\right|+q\left|a_{2}\right|\right)\right. \\
& \left.+\widehat{\lambda}_{2}\left(\frac{1}{q+1}+\sum_{j=1}^{p}\left|\beta_{j} \sigma_{j}^{q}\right|\right)\right], \\
\widehat{\Lambda}_{1}= & \widehat{\Lambda}-\frac{1}{\Gamma(q+1)},
\end{aligned}
$$

where

$$
\begin{aligned}
& \bar{\lambda}_{1}=\max _{t \in[0,1]}\left|\lambda_{1}(t)\right|=\frac{1}{|\Delta|}\left(\left|A_{2}\right|+\left|A_{1}\right|\right), \\
& \widehat{\lambda}_{2}=\max _{t \in[0,1]}\left|\widehat{\lambda}_{2}(t)\right|=\frac{1}{|\Delta|}\left(\left|\widehat{A}_{4}\right|+\left|A_{3}\right|\right) .
\end{aligned}
$$

With the aid of operator (5.2) and estimates (5.4) and (5.5), we can obtain the existence results for the fractional differential equation (1.1) supplemented with the boundary conditions (5.1) analogue to the ones for problem (1.1)-(1.2) established in Sect. 3.

\section{Multi-valued case}

In this section, we study the multi-valued analogue (inclusions case) of problem (1.1) given by

$$
\left\{\begin{array}{l}
{ }^{\mathrm{c}} D^{q} x(t) \in F(t, x(t)), \quad 0<t<1, m-1<q \leq m, m \geq 2, m \in N, \\
\int_{0}^{1} x(s) d s=\sum_{j=1}^{p} \beta_{j} x\left(\sigma_{j}\right), \quad x^{\prime}(0)=0, x^{\prime \prime}(0)=0, \ldots, x^{(m-2)}(0)=0, \\
a_{1} x(1)+a_{2}{ }^{\mathrm{c}} D^{q-1} x(1)=\sum_{i=1}^{n-2} \alpha_{i} \int_{\xi_{i}}^{\eta_{i}} x(s) d s
\end{array}\right.
$$

where $F: J \times \mathbb{R} \rightarrow 2^{\mathbb{R}} \backslash\{\emptyset\}(J=[0,1])$ and the other quantities are the same as defined in problem (1.1)-(1.2). We apply the Bohnenblust-Karlin fixed point theorem to prove the existence of solutions for problem (6.1).

For the convenience of the reader, we briefly recall some preliminary concepts about multi-valued maps [40, 41].

A multi-valued map $\mathcal{H}: X \rightarrow 2^{X}$ is (i) convex (closed) valued if $\mathcal{H}(x)$ is convex (closed) for all $x \in X$, where $(X,\|\cdot\|)$ is a Banach space; (ii) bounded on a bounded set if $\mathcal{H}(\mathbb{B})=$ $\bigcup_{x \in \mathbb{B}} \mathcal{H}(x)$ is bounded in $X$ for any bounded set $\mathbb{B}$ of $X$ (that is, $\sup _{x \in \mathbb{B}}\{\sup \{|y|: y \in \mathcal{H}(x)\}\}<$ $\infty$ ); (iii) upper semi-continuous (u.s.c.) on $X$ if, for each $x_{0} \in X$, the set $\mathcal{H}\left(x_{0}\right)$ is a nonempty closed subset of $X$ and if, for each open set $\mathbb{B}$ of $X$ containing $\mathcal{H}\left(x_{0}\right)$, there exists an open neighborhood $\mathcal{N}$ of $x_{0}$ such that $H(\mathcal{N}) \subseteq \mathbb{B}$; (iv) completely continuous if $\mathcal{H}(\mathbb{B})$ is relatively compact for every bounded subset $\mathbb{B}$ of $X$; (v) completely continuous with nonempty compact values, then $\mathcal{H}$ is u.s.c. if and only if $\mathcal{H}$ has a closed graph, that is, $x_{n} \rightarrow x_{*}, y_{n} \rightarrow y_{*}$, $y_{n} \in \mathcal{H}\left(x_{n}\right)$ imply $y_{*} \in H\left(x_{*}\right)$.

A multi-valued map $\mathcal{H}: X \rightarrow 2^{X}$ has a fixed point if there is $x \in X$ such that $x \in \mathcal{H}(x)$.

In the sequel, we denote by $B C C(X)$ the set of all nonempty bounded, closed, and convex subsets of $X$ and $L^{1}(J, \mathbb{R})$ denotes the Banach space of functions $x: J \rightarrow \mathbb{R}$ which are Lebesgue integrable and normed by $\|x\|_{L^{1}}=\int_{0}^{1}|x(t)| d t$. 
Now we state the assumptions needed in the forthcoming analysis.

$\left(\mathrm{M}_{1}\right)$ Let $F: J \times \mathbb{R} \rightarrow B C C(\mathbb{R}) ;(t, x) \rightarrow f(t, x)$ be measurable with respect to $t$ for each $x \in \mathbb{R}$, u.s.c. with respect to $x$ for a.e. $t \in J$, and for each fixed $x \in \mathbb{R}$, the set $S_{F, x}:=$ $\left\{f \in L^{1}(J, \mathbb{R}): f(t) \in F(t, x)\right.$ for a.e. $\left.t \in J\right\}$ is nonempty.

$\left(\mathrm{M}_{2}\right)$ For each $\rho>0$, there exists a function $p_{\rho} \in L^{1}\left(J, \mathbb{R}^{+}\right)$such that $\|F(t, x)\|=\sup \{|v|$ : $v(t) \in F(t, x)\} \leq p_{\rho}(t)$ for each $(t, x) \in J \times \mathbb{R}$ with $|x| \leq \rho$, and

$$
\liminf _{\rho \rightarrow+\infty}\left(\frac{\int_{0}^{1} p_{\rho}(t) d t}{\rho}\right)=\mu<\infty
$$

The proof of our main result is based on the following lemmas.

Lemma 6.1 (Bohnenblust-Karlin [42]) Let $D \subset X$ be nonempty bounded, closed, and convex. Let $\mathcal{H}: D \rightarrow 2^{X} \backslash\{0\}$ be u.s.c. with closed, convex values such that $\mathcal{H}(D) \subset D$ and $\overline{\mathcal{H}(D)}$ is compact. Then $\mathcal{H}$ has a fixed point.

Lemma 6.2 ([43]) Let $F$ be a multi-valued map satisfying condition $\left(M_{1}\right)$, and $\phi$ is linear continuous from $L^{1}(J, \mathbb{R}) \rightarrow C(J, \mathbb{R})$. Then the operator $\phi \circ S_{F}: C(J, \mathbb{R}) \rightarrow B C C(C(J, \mathbb{R}))$, $x \mapsto\left(\phi \circ S_{F}\right)(x)=\phi\left(S_{F, x}\right)$ is a closed graph operator in $C(J, \mathbb{R}) \times C(J, \mathbb{R})$.

Theorem 6.3 Assume that $\left(\mathrm{M}_{1}\right)$ and $\left(\mathrm{M}_{2}\right)$ hold and that

$$
\mu \Lambda_{2}<1
$$

where $\mu$ is given by (6.2) and

$$
\Lambda_{2}=\frac{1}{\Gamma(q)}\left\{1+\bar{\lambda}_{1}\left(\sum_{i=1}^{n-2}\left|\alpha_{i} \eta_{i}^{q}\right|+\left|a_{1}\right|+\left|a_{2}\right| \Gamma(q)\right)+\bar{\lambda}_{2}\left(1+\sum_{j=1}^{p}\left|\beta_{j} \sigma_{j}^{q-1}\right|\right)\right\} .
$$

Then there exists at least one solution for problem (6.1) on J.

Proof We first transform problem (6.1) into a fixed point problem by introducing a multivalued map $\Psi: C(J, \mathbb{R}) \rightarrow 2^{C(J, \mathbb{R})}$ as follows:

$$
\begin{aligned}
\Psi(x)= & \left\{h \in C[0,1]: h(t)=\int_{0}^{t} \frac{(t-s)^{q-1}}{\Gamma(q)} f(s) d s\right. \\
& -\lambda_{1}(t)\left[\sum_{i=1}^{n-2} \alpha_{i} \int_{\xi_{i}}^{\eta_{i}}\left(\int_{0}^{s} \frac{(s-u)^{q-1}}{\Gamma(q)} f(u) d u\right) d s\right. \\
& \left.-a_{1} \int_{0}^{1} \frac{(1-s)^{q-1}}{\Gamma(q)} f(s) d s-a_{2} \int_{0}^{1} f(s) d s\right] \\
& -\lambda_{2}(t)\left[\int_{0}^{1}\left(\int_{0}^{s} \frac{(s-u)^{q-1}}{\Gamma(q)} f(u) d u\right) d s\right. \\
& \left.\left.-\sum_{j=1}^{p} \beta_{j} \int_{0}^{\sigma_{j}} \frac{\left(\sigma_{j}-s\right)^{q-1}}{\Gamma(q)} f(s) d s\right], f \in S_{F, x}\right\} .
\end{aligned}
$$


It will be shown that the operator $\Psi$ satisfies the hypothesis of Lemma 6.1 and hence it will have a fixed point. This will establish the existence of a solution for problem (6.1).

Let us first show that $\Psi(x)$ is convex for each $x \in C(J, \mathbb{R})$. For $h_{1}, h_{2} \in \Psi(x)$, there exist $f_{1}, f_{2} \in S_{F, x}$ such that, for each $t \in J$, we get

$$
\begin{aligned}
h_{i}(t)= & \int_{0}^{t} \frac{(t-s)^{q-1}}{\Gamma(q)} f_{i}(s) d s \\
& -\lambda_{1}(t)\left[\sum_{i=1}^{n-2} \alpha_{i} \int_{\xi_{i}}^{\eta_{i}}\left(\int_{0}^{s} \frac{(s-u)^{q-1}}{\Gamma(q)} f_{i}(u) d u\right) d s\right. \\
& \left.-a_{1} \int_{0}^{1} \frac{(1-s)^{q-1}}{\Gamma(q)} f_{i}(s) d s-a_{2} \int_{0}^{1} f_{i}(s) d s\right] \\
& -\lambda_{2}(t)\left[\int_{0}^{1}\left(\int_{0}^{s} \frac{(s-u)^{q-1}}{\Gamma(q)} f_{i}(u) d u\right) d s-\sum_{j=1}^{p} \beta_{j} \int_{0}^{\sigma_{j}} \frac{\left(\sigma_{j}-s\right)^{q-1}}{\Gamma(q)} f_{i}(s) d s\right], \\
& i=1,2 .
\end{aligned}
$$

For $0 \leq \sigma \leq 1$ and each $t \in J$, we obtain

$$
\begin{aligned}
& {\left[\sigma h_{1}+(1-\sigma) h_{2}\right](t)} \\
& =\int_{0}^{t} \frac{(t-s)^{q-1}}{\Gamma(q)}\left[\sigma f_{1}(s)+(1-\sigma) f_{2}(s)\right] d s \\
& \quad-\lambda_{1}(t)\left[\sum_{i=1}^{n-2} \alpha_{i} \int_{\xi_{i}}^{\eta_{i}}\left(\int_{0}^{s} \frac{(s-u)^{q-1}}{\Gamma(q)}\left[\sigma f_{1}(s)+(1-\sigma) f_{2}(s)\right] d u\right) d s\right. \\
& \left.\quad-a_{1} \int_{0}^{1} \frac{(1-s)^{q-1}}{\Gamma(q)}\left[\sigma f_{1}(s)+(1-\sigma) f_{2}(s)\right] d s-a_{2} \int_{0}^{1}\left[\sigma f_{1}(s)+(1-\sigma) f_{2}(s)\right] d s\right] \\
& \quad-\lambda_{2}(t)\left[\int_{0}^{1}\left(\int_{0}^{s} \frac{(s-u)^{q-1}}{\Gamma(q)}\left[\sigma f_{1}(s)+(1-\sigma) f_{2}(s)\right] d u\right) d s\right. \\
& \left.\quad-\sum_{j=1}^{p} \beta_{j} \int_{0}^{\sigma_{j}} \frac{\left(\sigma_{j}-s\right)^{q-1}}{\Gamma(q)}\left[\sigma f_{1}(s)+(1-\sigma) f_{2}(s)\right] d s\right] .
\end{aligned}
$$

As $S_{F, x}$ is convex ( $F$ has convex values), therefore one can infer that $\sigma h_{1}+(1-\sigma) h_{2} \in \Psi(x)$.

In the next step, we show that there exists a positive number $\rho$ such that $\Psi\left(B_{\rho}\right) \subseteq B_{\rho}$, where $B_{\rho}=\{x \in C(J, \mathbb{R}):\|x\| \leq \rho\}$. For each positive constant $\rho$, observe that $B_{\rho}$ is a bounded closed convex set in $C(J, \mathbb{R})$. If it is not true, then we can find a function $x_{\rho} \in B_{\rho}, h_{\rho} \in \Psi\left(x_{\rho}\right)$ with $\left\|\Psi\left(x_{\rho}\right)\right\|>\rho$ for each positive number $\rho$ such that

$$
\begin{aligned}
h_{\rho}(t)= & \int_{0}^{t} \frac{(t-s)^{q-1}}{\Gamma(q)} f_{\rho}(s) d s \\
& -\lambda_{1}(t)\left[\sum_{i=1}^{n-2} \alpha_{i} \int_{\xi_{i}}^{\eta_{i}}\left(\int_{0}^{s} \frac{(s-u)^{q-1}}{\Gamma(q)} f_{\rho}(u) d u\right) d s\right.
\end{aligned}
$$




$$
\begin{aligned}
& \left.-a_{1} \int_{0}^{1} \frac{(1-s)^{q-1}}{\Gamma(q)} f_{\rho}(s) d s-a_{2} \int_{0}^{1} f_{\rho}(s) d s\right] \\
& -\lambda_{2}(t)\left[\int_{0}^{1}\left(\int_{0}^{s} \frac{(s-u)^{q-1}}{\Gamma(q)} f_{\rho}(u) d u\right) d s-\sum_{j=1}^{p} \beta_{j} \int_{0}^{\sigma_{j}} \frac{\left(\sigma_{j}-s\right)^{q-1}}{\Gamma(q)} f_{\rho}(s) d s\right]
\end{aligned}
$$

for some $f_{\rho} \in S_{F, x_{\rho}}$. On the other hand, using $\left(M_{2}\right)$, we find that

$$
\begin{aligned}
\rho< & \left\|\Psi\left(x_{\rho}\right)\right\| \\
\leq & \int_{0}^{t} \frac{(t-s)^{q-1}}{\Gamma(q)} p_{\rho}(s) d s \\
& +\left|\lambda_{1}(t)\right|\left[\sum_{i=1}^{n-2}\left|\alpha_{i}\right| \int_{\xi_{i}}^{\eta_{i}}\left(\int_{0}^{s} \frac{(s-u)^{q-1}}{\Gamma(q)} p_{\rho}(u) d u\right) d s\right. \\
& \left.+\left|a_{1}\right| \int_{0}^{1} \frac{(1-s)^{q-1}}{\Gamma(q)} p_{\rho}(s) d s+\left|a_{2}\right| \int_{0}^{1} p_{\rho}(s) d s\right] \\
& +\left|\lambda_{2}(t)\right|\left[\int_{0}^{1}\left(\int_{0}^{s} \frac{(s-u)^{q-1}}{\Gamma(q)} p_{\rho}(u) d u\right) d s+\sum_{j=1}^{p}\left|\beta_{j}\right| \int_{0}^{\sigma_{j}} \frac{\left(\sigma_{j}-s\right)^{q-1}}{\Gamma(q)} p_{\rho}(s) d s\right] \\
\leq & \Lambda_{2} \int_{0}^{1} p_{\rho}(s) d s,
\end{aligned}
$$

where $\Lambda_{2}$ is given by (6.4). Dividing both sides of the above inequality by $\rho$ and taking the lower limit as $\rho \rightarrow \infty$ together with notation (6.2), we obtain

$$
1 \leq \mu \Lambda_{2}
$$

which contradicts (6.3). Consequently, there exists a positive number $\rho_{1}$ such that $\Psi\left(B_{\rho_{1}}\right) \subseteq B_{\rho_{1}}$.

Now we show that $\Psi\left(B_{\rho}\right)$ is equi-continuous. For $0<t_{1}<t_{2}<1, x \in B_{\rho}$, and $h \in \Psi(x)$, there exists $f \in S_{F, x}$ such that, for each $t \in J$, we have

$$
\begin{aligned}
h(t)= & \int_{0}^{t} \frac{(t-s)^{q-1}}{\Gamma(q)} f(s) d s \\
& -\lambda_{1}(t)\left[\sum_{i=1}^{n-2} \alpha_{i} \int_{\xi_{i}}^{\eta_{i}}\left(\int_{0}^{s} \frac{(s-u)^{q-1}}{\Gamma(q)} f(u) d u\right) d s\right. \\
& \left.-a_{1} \int_{0}^{1} \frac{(1-s)^{q-1}}{\Gamma(q)} f(s) d s-a_{2} \int_{0}^{1} f(s) d s\right] \\
& -\lambda_{2}(t)\left[\int_{0}^{1}\left(\int_{0}^{s} \frac{(s-u)^{q-1}}{\Gamma(q)} f(u) d u\right) d s-\sum_{j=1}^{p} \beta_{j} \int_{0}^{\sigma_{j}} \frac{\left(\sigma_{j}-s\right)^{q-1}}{\Gamma(q)} f(s) d s\right]
\end{aligned}
$$

and

$$
\begin{aligned}
\left|h\left(t_{2}\right)-h\left(t_{1}\right)\right| \leq & \int_{0}^{t_{1}} \frac{\left[\left(t_{2}-s\right)^{q-1}-\left(t_{1}-s\right)^{q-1}\right]}{\Gamma(q)} p_{\rho}(s) d s+\int_{t_{1}}^{t_{2}} \frac{\left(t_{2}-s\right)^{q-1}}{\Gamma(q)} p_{\rho}(s) d s \\
& +\left|A_{1}\left(t_{2}^{m-1}-t_{1}^{m-1}\right)\right|\left[\sum_{i=1}^{n-2}\left|\alpha_{i}\right| \int_{\xi_{i}}^{\eta_{i}}\left(\int_{0}^{s} \frac{(s-u)^{q-1}}{\Gamma(q)} p_{\rho}(u) d u\right) d s\right.
\end{aligned}
$$




$$
\begin{aligned}
& \left.+\left|a_{1}\right| \int_{0}^{1} \frac{(1-s)^{q-1}}{\Gamma(q)} p_{\rho}(s) d s+\left|a_{2}\right| \int_{0}^{1} p_{\rho}(s) d s\right] \\
& +\left|A_{3}\left(t_{2}^{m-1}-t_{1}^{m-1}\right)\right|\left[\int_{0}^{1}\left(\int_{0}^{s} \frac{(s-u)^{q-1}}{\Gamma(q)} p_{\rho}(u) d u\right) d s\right. \\
& \left.+\sum_{j=1}^{p}\left|\beta_{j}\right| \int_{0}^{\sigma_{j}} \frac{\left(\sigma_{j}-s\right)^{q-1}}{\Gamma(q)} p_{\rho}(s) d s\right] .
\end{aligned}
$$

It is obvious that the right-hand side of the above inequality tends to zero independently of $x \in B_{\rho}$ as $t_{2} \rightarrow t_{1}$. Hence $\Psi$ is equi-continuous. Since $\Psi$ satisfies the above three conditions, it follows by the Ascoli-Arzela theorem that $\Psi$ is a compact multi-valued map.

Next, we show that the operator $\Psi$ has a closed graph. Let $x_{n} \rightarrow x_{*}, h_{n} \in \Psi\left(x_{n}\right)$ and $h_{n} \rightarrow h_{*}$. We will show that $h_{*} \in \Psi\left(x_{*}\right)$. For $h_{n} \in \Psi\left(x_{n}\right)$, we can find $f_{n} \in S_{F, x_{n}}$ for each $t \in J$ such that

$$
\begin{aligned}
h_{n}(t)= & \int_{0}^{t} \frac{(t-s)^{q-1}}{\Gamma(q)} f_{n}(s) d s \\
& -\lambda_{1}(t)\left[\sum_{i=1}^{n-2} \alpha_{i} \int_{\xi_{i}}^{\eta_{i}}\left(\int_{0}^{s} \frac{(s-u)^{q-1}}{\Gamma(q)} f_{n}(u) d u\right) d s\right. \\
& \left.-a_{1} \int_{0}^{1} \frac{(1-s)^{q-1}}{\Gamma(q)} f_{n}(s) d s-a_{2} \int_{0}^{1} f_{n}(s) d s\right] \\
& -\lambda_{2}(t)\left[\int_{0}^{1}\left(\int_{0}^{s} \frac{(s-u)^{q-1}}{\Gamma(q)} f_{n}(u) d u\right) d s-\sum_{j=1}^{p} \beta_{j} \int_{0}^{\sigma_{j}} \frac{\left(\sigma_{j}-s\right)^{q-1}}{\Gamma(q)} f_{n}(s) d s\right] .
\end{aligned}
$$

Thus, for each $t \in J$, we have to show that there exists $f_{*} \in S_{F, x_{*}}$ such that

$$
\begin{aligned}
h_{*}(t)= & \int_{0}^{t} \frac{(t-s)^{q-1}}{\Gamma(q)} f_{*}(s) d s \\
& -\lambda_{1}(t)\left[\sum_{i=1}^{n-2} \alpha_{i} \int_{\xi_{i}}^{\eta_{i}}\left(\int_{0}^{s} \frac{(s-u)^{q-1}}{\Gamma(q)} f_{*}(u) d u\right) d s\right. \\
& \left.-a_{1} \int_{0}^{1} \frac{(1-s)^{q-1}}{\Gamma(q)} f_{*}(s) d s-a_{2} \int_{0}^{1} f_{*}(s) d s\right] \\
& -\lambda_{2}(t)\left[\int_{0}^{1}\left(\int_{0}^{s} \frac{(s-u)^{q-1}}{\Gamma(q)} f_{*}(u) d u\right) d s-\sum_{j=1}^{p} \beta_{j} \int_{0}^{\sigma_{j}} \frac{\left(\sigma_{j}-s\right)^{q-1}}{\Gamma(q)} f_{*}(s) d s\right] .
\end{aligned}
$$

Considering the continuous linear operator $\phi: L^{1}(J, \mathbb{R}) \rightarrow C(J, \mathbb{R})$, we get

$$
\begin{aligned}
f \mapsto \phi(f)(t) & =\int_{0}^{t} \frac{(t-s)^{q-1}}{\Gamma(q)} f(s) d s \\
& -\lambda_{1}(t)\left[\sum_{i=1}^{n-2} \alpha_{i} \int_{\xi_{i}}^{\eta_{i}}\left(\int_{0}^{s} \frac{(s-u)^{q-1}}{\Gamma(q)} f(u) d u\right) d s\right.
\end{aligned}
$$




$$
\begin{aligned}
& \left.-a_{1} \int_{0}^{1} \frac{(1-s)^{q-1}}{\Gamma(q)} f(s) d s-a_{2} \int_{0}^{1} f(s) d s\right] \\
& -\lambda_{2}(t)\left[\int_{0}^{1}\left(\int_{0}^{s} \frac{(s-u)^{q-1}}{\Gamma(q)} f(u) d u\right) d s-\sum_{j=1}^{p} \beta_{j} \int_{0}^{\sigma_{j}} \frac{\left(\sigma_{j}-s\right)^{q-1}}{\Gamma(q)} f(s) d s\right] .
\end{aligned}
$$

Note that

$$
\begin{aligned}
h_{n}(t)-h_{*}(t)= & \int_{0}^{t} \frac{(t-s)^{q-1}}{\Gamma(q)}\left(f_{n}(s)-f_{*}(s)\right) d s \\
& -\lambda_{1}(t)\left[\sum_{i=1}^{n-2} \alpha_{i} \int_{\xi_{i}}^{\eta_{i}}\left(\int_{0}^{s} \frac{(s-u)^{q-1}}{\Gamma(q)}\left(f_{n}(u)-f_{*}(u)\right) d u\right) d s\right. \\
& \left.-a_{1} \int_{0}^{1} \frac{(1-s)^{q-1}}{\Gamma(q)}\left(f_{n}(s)-f_{*}(s)\right) d s-a_{2} \int_{0}^{1}\left(f_{n}(s)-f_{*}(s)\right) d s\right] \\
& -\lambda_{2}(t)\left[\int_{0}^{1}\left(\int_{0}^{s} \frac{(s-u)^{q-1}}{\Gamma(q)}\left(f_{n}(u)-f_{*}(u)\right) d u\right) d s\right. \\
& \left.-\sum_{j=1}^{p} \beta_{j} \int_{0}^{\sigma_{j}} \frac{\left(\sigma_{j}-s\right)^{q-1}}{\Gamma(q)}\left(f_{n}(s)-f_{*}(s)\right) d s\right] \\
\rightarrow & 0 \quad \text { as } n \rightarrow \infty .
\end{aligned}
$$

In consequence, it follows by Lemma 6.2 that $\phi \circ S_{F}$ is a closed graph operator and that $h_{n}(t) \in \phi\left(S_{F, x_{n}}\right)$ as $x_{n} \rightarrow x_{*}$; therefore, Lemma 6.2 yields

$$
\begin{aligned}
h_{*}(t)= & \int_{0}^{t} \frac{(t-s)^{q-1}}{\Gamma(q)} f_{*}(s) d s \\
& -\lambda_{1}(t)\left[\sum_{i=1}^{n-2} \alpha_{i} \int_{\xi_{i}}^{\eta_{i}}\left(\int_{0}^{s} \frac{(s-u)^{q-1}}{\Gamma(q)} f_{*}(u) d u\right) d s\right. \\
& \left.-a_{1} \int_{0}^{1} \frac{(1-s)^{q-1}}{\Gamma(q)} f_{*}(s) d s-a_{2} \int_{0}^{1} f_{*}(s) d s\right] \\
& -\lambda_{2}(t)\left[\int_{0}^{1}\left(\int_{0}^{s} \frac{(s-u)^{q-1}}{\Gamma(q)} f_{*}(u) d u\right) d s-\sum_{j=1}^{p} \beta_{j} \int_{0}^{\sigma_{j}} \frac{\left(\sigma_{j}-s\right)^{q-1}}{\Gamma(q)} f_{*}(s) d s\right] .
\end{aligned}
$$

Hence, we deduce that $\Psi$ is a compact multi-valued map, u.s.c. with convex closed values. Thus, the hypothesis of Lemma 6.1 holds true, and consequently its conclusion implies that the operator $\Psi$ has a fixed point $x$, which is indeed a solution of problem (6.1). This completes the proof.

Remark 6.4 For $a_{1}=0, a_{2}=1$, our results obtained in Sects. 3 and 4 reduce to the ones with the last condition in (1.2) of the form: ${ }^{\mathrm{c}} D^{q-1} x(1)=\sum_{i=1}^{n-2} \alpha_{i} \int_{\xi_{i}}^{\eta_{i}} x(s) d s$ and $x^{\prime}(1)=$ $\sum_{i=1}^{n-2} \alpha_{i} \int_{\xi_{i}}^{\eta_{i}} x(s) d s$, respectively, which are indeed new. On the other hand, we obtain the new results associated with the last condition in (1.2) of the form: $x(1)=\sum_{i=1}^{n-2} \alpha_{i} \int_{\xi_{i}}^{\eta_{i}} x(s) d s$ by fixing $a_{1}=1, a_{2}=0$. 


\section{Acknowledgements}

This project was funded by the Deanship of Scientific Research (DSR), King Abdulaziz University, Jeddah, Saudi Arabia under grant no. (KEP-MSC-10-130-39). The authors, therefore, acknowledge with thanks DSR for technical and financial support. We also thank the editor and the reviewers for their useful remarks on our paper.

\section{Funding}

This project was funded by the Deanship of Scientific Research (DSR), King Abdulaziz University, Jeddah, Saudi Arabia under grant no. (KEP-MSc-10-130-39).

\section{Abbreviations}

Not applicable.

\section{Availability of data and materials}

Not applicable.

\section{Competing interests}

The authors declare that they have no competing interests.

\section{Authors' contributions}

Each of the authors, SS, AA, BA, and MA, contributed equally to each part of this work. All authors read and approved the final manuscript.

\section{Publisher's Note}

Springer Nature remains neutral with regard to jurisdictional claims in published maps and institutional affiliations.

Received: 30 July 2018 Accepted: 30 September 2018 Published online: 24 October 2018

\section{References}

1. Kilbas, A.A., Srivastava, H.M., Trujillo, J..: Theory and Applications of Fractional Differential Equations. North-Holland Mathematics Studies, vol. 204. Elsevier, Amsterdam (2006)

2. Lakshmikantham, V., Leela, S., Vasundhara Devi, J.: Theory of Fractional Dynamic Systems. Cambridge Academic Publishers, Cambridge (2009)

3. Zhou, Y:: Basic Theory of Fractional Differential Equations. World Scientific, Hackensack (2014)

4. Ahmad, B., Alsaedi, A., Ntouyas, S.K., Tariboon, J.: Hadamard-Type Fractional Differential Equations, Inclusions and Inequalities. Springer, Berlin (2017)

5. Magin, R.L.: Fractional Calculus in Bioengineering. Begell House Publishers, Danbury (2006)

6. Sabatier, J., Agrawal, O.P., Machado, J.A.T. (eds.): Advances in Fractional Calculus: Theoretical Developments and Applications in Physics and Engineering. Springer, Dordrecht (2007)

7. Klafter, J., Lim, S.C., Metzler, R. (eds.): Fractional Dynamics in Physics. World Scientific, Singapore (2011)

8. Petras, I., Magin, R.L.: Simulation of drug uptake in a two compartmental fractional model for a biological system. Commun. Nonlinear Sci. Numer. Simul. 16, 4588-4595 (2011)

9. Ding, Y., Wang, Z., Ye, H.: Optimal control of a fractional-order HIV-immune system with memory. IEEE Trans. Control Syst. Technol. 20, 763-769 (2012)

10. Javidi, M., Ahmad, B.: Dynamic analysis of time fractional order phytoplankton-toxic phytoplankton-zooplankton system. Ecol. Model. 318, 8-18 (2015)

11. Povstenko, Y.Z: Fractional Thermoelasticity. Springer, New York (2015)

12. Carvalho, A., Pinto, C.M.A.: A delay fractional order model for the co-infection of malaria and HIV/AIDS. Int. J. Dyn. Control 5, 168-186 (2017)

13. Whyburn, W.M.: Differential equations with general boundary conditions. Bull. Am. Math. Soc. 48, 692-704 (1942)

14. Bitsadze, A.V., Samarskii, A.A.: Some elementary generalizations of linear elliptic boundary value problems. Sov. Math. Dokl. 10, 398-400 (1969)

15. Ahmad, B., Alsaedi, A., Alghamdi, B.S.: Analytic approximation of solutions of the forced Duffing equation with integral boundary conditions. Nonlinear Anal., Real World Appl. 9, 1727-1740 (2008)

16. Čiegis, R., Bugajev, A.: Numerical approximation of one model of the bacterial self-organization. Nonlinear Anal., Model. Control 17, 253-270 (2012)

17. Wang, J.R., Zhou, Y., Feckan, M.: On recent developments in the theory of boundary value problems for impulsive fractional differential equations. Comput. Math. Appl. 64, 3008-3020 (2012)

18. Graef, J.R., Kong, L.: Existence of positive solutions to a higher order singular boundary value problem with fractional q-derivatives. Fract. Calc. Appl. Anal. 16, 695-708 (2013)

19. O'Regan, D., Stanek, S.: Fractional boundary value problems with singularities in space variables. Nonlinear Dyn. 71, 641-652 (2013)

20. Alsaedi, A., Ntouyas, S.K., Agarwal, R.P., Ahmad, B.: A nonlocal multi-point multi-term fractional boundary value problem with Riemann-Liouville type integral boundary conditions involving two indices. Adv. Differ. Equ. 2013, 369 (2013)

21. Zhai, C., Xu, L.: Properties of positive solutions to a class of four-point boundary value problem of Caputo fractional differential equations with a parameter. Commun. Nonlinear Sci. Numer. Simul. 19, 2820-2827 (2014)

22. Henderson, J., Kosmatov, N.: Eigenvalue comparison for fractional boundary value problems with the Caputo derivative. Fract. Calc. Appl. Anal. 17, 872-880 (2014)

23. Li, B., Sun, S., Li, Y., Zhao, P.: Multi-point boundary value problems for a class of Riemann-Liouville fractional differential equations. Adv. Differ. Equ. 2014, 151 (2014) 
24. Zhang, L., Ahmad, B., Wang, G.: Successive iterations for positive extremal solutions of nonlinear fractional differential equations on a half line. Bull. Aust. Math. Soc. 91, 116-128 (2015)

25. Henderson, J., Luca, R.: Nonexistence of positive solutions for a system of coupled fractional boundary value problems. Bound. Value Probl. 2015, 138 (2015)

26. Ntouyas, S.K., Etemad, S.: On the existence of solutions for fractional differential inclusions with sum and integral boundary conditions. Appl. Math. Comput. 266, 235-243 (2015)

27. Qarout, D., Ahmad, B., Alsaedi, A.: Existence theorems for semilinear Caputo fractional differential equations with nonlocal discrete and integral boundary conditions. Fract. Calc. Appl. Anal. 19, 463-479 (2016)

28. Ahmad, B., Ntouyas, S.K., Agarwal, R.P., Alsaedi, A.: Existence results for sequential fractional integro-differential equations with nonlocal multi-point and strip conditions. Bound. Value Probl. 2016, 205 (2016)

29. Zou, Y., He, G.: On the uniqueness of solutions for a class of fractional differential equations. Appl. Math. Lett. 74, 68-73 (2017)

30. Agarwal, R.P., Ahmad, B., Nieto, J.J.: Fractional differential equations with nonlocal (parametric type) anti-periodic boundary conditions. Filomat 31, 1207-1214 (2017)

31. Yang, X.-J., Gao, F., Machado, J.A.T., Baleanu, D.: A new fractional derivative involving the normalized sinc function without singular kernel. Eur. Phys. J. Spec. Top. 226, 3567-3575 (2017)

32. Yang, X.-J., Srivastava, H.M., Machado, J.A.T.: A new fractional derivative without singular kernel: application to the modelling of the steady heat flow. Therm. Sci. 20, 753-756 (2016)

33. Agarwal, R.P., Ahmad, B., Garout, D., Alsaedi, A.: Existence results for coupled nonlinear fractional differential equations equipped with nonlocal coupled flux and multi-point boundary conditions. Chaos Solitons Fractals 102, 149-161 (2017)

34. Xu, M., Han, Z.: Positive solutions for integral boundary value problem of two-term fractional differential equations. Bound. Value Probl. 2018, 100 (2018)

35. Wang, G., Pei, K., Agarwal, R.P., Zhang, L., Ahmad, B.: Nonlocal Hadamard fractional boundary value problem with Hadamard integral and discrete boundary conditions on a half-line. J. Comput. Appl. Math. 343, 230-239 (2018)

36. Agarwal, R.P., Hristova, S., O'Regan, D.: Iterative techniques for the initial value problem for Caputo fractional differential equations with non-instantaneous impulses. Appl. Math. Comput. 334, 407-421 (2018)

37. Ahmad, B., Luca, R.: Existence of solutions for a system of fractional differential equations with coupled nonlocal boundary conditions. Fract. Calc. Appl. Anal. 21, 423-441 (2018)

38. Granas, A., Dugundji, J.: Fixed Point Theory. Springer, New York (2003)

39. Krasnoselskii, M.A.: Two remarks on the method of successive approximations. Usp. Mat. Nauk 10, 123-127 (1955)

40. Deimling, K:: Multivalued Differential Equations. de Gruyter, Berlin (1992)

41. Hu, S., Papageorgiou, N.: Handbook of Multivalued Analysis. Kluwer Academic, Dordrecht (1997)

42. Bohnenblust, H.F., Karlin, S.: On a theorem of Ville. In: Contributions to the Theory of Games, vol. I, pp. 155-160. Princeton Univeristy Press, Princeton (1950)

43. Lasota, A., Opial, Z:: An application of the Kakutani-Ky Fan theorem in the theory of ordinary differential equations. Bull. Acad. Pol. Sci., Sér. Sci. Math. Astron. Phys. 13, 781-786 (1965)

\section{Submit your manuscript to a SpringerOpen ${ }^{\circ}$ journal and benefit from:}

- Convenient online submission

- Rigorous peer review

- Open access: articles freely available online

- High visibility within the field

- Retaining the copyright to your article

Submit your next manuscript at $\gg$ springeropen.com 